Artigo Original

Original Article

Marizete Ilha Ceron ${ }^{1}$

Marileda Barichello Gubiani ${ }^{1}$

Camila Rosa de Oliveira²

Márcia Keske-Soares ${ }^{3}$

Descritores

Testes de articulação da fala

Transtornos da articulação

Avaliação

Criança

Fala

Keywords

Speech articulation tests

Articulation disorders

Evaluation

Child

Speech

Endereço para correspondência:

Marizete Ilha Ceron

Rua Bem-te-vi, 215, Bairro JK,

Santa Maria (RS)

CEP: 97035-130

E-mail: marizeteceron@hotmail.com

Recebido em: Abril 08, 2019.

Aceito em: Setembro 10, 2019.

\section{Instrumento de Avaliação Fonológica (INFONO): estudo piloto}

\author{
Phonological Assessment Instrument (INFONO): \\ A pilot study
}

\begin{abstract}
Purpose: To analyze the recognizability and effectiveness of items in the Phonological Assessment Tool (Instrumento de Avaliação Fonológica - INFONO) at eliciting target words, as well as to evaluate the internal consistency of the instrument and compare performance between genders, school types and typical vs. atypical phonological development. Method: Participants were 48 children ( $n=26$ with typical phonological development and $n=22$ with atypical development) categorized by age ( 6 age groups ranging from 3 years and 8 years 11 months), type of school (public vs. private) and gender (male vs. female). Data were collected by the spontaneous naming task of the INFONO. Recognition rates, scores, recognition difficulties and internal consistency were examined in 116 items. Performance in a final set of 84 items was also compared between genders, school types and typical/ atypical phonological development. Results: Most target words achieved high recognition rates were considered suitable for use in the INFONO. Some images had to be redesigned to facilitate the spontaneous production of target words, while other items were excluded from the instrument altogether. The instrument demonstrated excellent internal consistency. There were no statistically significant differences between genders and school types, though differences were observed between typically and atypically developing children. Conclusion: The images in the INFONO were successfully recognized by participants and were effective at eliciting the target words. The final set of items contained the minimum number of target words which would allow for an assessment of all phonemes in Brazilian Portuguese in different word and syllable positions, and these items presented excellent internal consistency.
\end{abstract}

Trabalho realizado no Departamento de Fonoaudiologia, Curso de Pós-Graduação em Distúrbios da Comunicação Humana, Universidade Federal de Santa Maria - UFSM - Santa Maria (RS), Brasil.

${ }^{1}$ Secretaria Municipal de Santa Cruz do Sul - Santa Cruz do Sul (RS), Brasil.

${ }^{2}$ Curso de Psicologia, Faculdade Meridional do Rio Grande do Sul - IMED - Passo Fundo (RS), Brasil.

${ }^{3}$ Departamento de Fonoaudiologia, Universidade Federal de Santa Maria - UFSM - Santa Maria (RS),

Brasil.

Conflito de interesses: Nada a declarar.

Fonte de financiamento: Programa Pesquisador Gaúcho $(\mathrm{PqG})$ - Fundação de Amparo à Pesquisa do Estado do Rio Grande do Sul (FAPERGS) (processo número 11/2184-8). 


\section{INTRODUÇÃO}

A habilidade de produzir os sons da fala começa na infância, havendo expansão desta habilidade na idade pré-escolar. O número de omissões e substituições de fonemas durante o desenvolvimento fonológico típico deve decrescer com o aumento da idade cronológica, acontecendo um rápido declínio nos primeiros anos (entre dois e três anos), e pouca ou nenhuma mudança nos últimos anos (entre quatro e seis anos) ${ }^{(1,2)}$.

O desenvolvimento fonológico inicia-se com a emissão dos primeiros sons e acontece de forma progressiva até a idade aproximada de cinco $\operatorname{anos}^{(3)}$, momento em que, em geral, há a aquisição completa dos sons da fala. A cronologia de aquisição e desenvolvimento das classes de sons é: plosivas e nasais; fricativas, e líquidas ${ }^{(4)}$. Em relação à estrutura da sílaba, a ordem de desenvolvimento no Português Brasileiro (PB) é Consoante-Vogal (CV), Consoante-Vogal-Vogal (CVV), Consoante-Vogal-Consoante (CVC) e, por fim, ConsoanteConsoante-Vogal $(\mathrm{CCV})^{(4)}$.

Para que este desenvolvimento ocorra de modo adequado, é necessário que a criança aprenda tanto os movimentos articulatórios da produção dos sons (aspectos fonéticos) quanto os aspectos organizacionais ou estruturais do inventário de fonemas da língua (aspectos fonológicos) ${ }^{(5,6)}$. Para algumas crianças, este desenvolvimento parece ser complexo e elas podem apresentar um e/ou outro destes aspectos alterados.

Em relação ao aspecto fonológico, frequentemente encontram-se crianças que apresentam omissão e/ou substituição de fonemas, especialmente consoantes, e redução da estrutura de encontro consonantal, em idade que estas alterações não deveriam mais acontecer ${ }^{(5)}$. Quando isso ocorre, tem-se o desvio fonológico, que é frequentemente alvo de pesquisas e apresenta alto índice de ocorrência na infância ${ }^{(5,7-10)}$.

As crianças com desvio fonológico, ao tentarem produzir algumas palavras, encontrarão dificuldades, pois não apresentam, em seu inventário fonológico, fonemas suficientes para produzilos de forma correta. Isto torna a fala de difícil compreensão, especialmente para aquelas que apresentam maior gravidade, como, por exemplo, o desvio fonológico de grau moderado a grave ${ }^{(6,10)}$. A fala, quando é ininteligível, pode ter impactos adversos na comunicação e interação social ${ }^{(5,6,9,10)}$. Além disso, o desvio fonológico pode influenciar no desempenho escolar, uma vez que a fala é essencial para o processo de alfabetização, e os erros de fala poderão ser reproduzidos para a escrita. Dessa forma, são fundamentais o diagnóstico e a intervenção precoces para evitar o agravamento do problema ${ }^{(5)}$.

$\mathrm{Na}$ literatura internacional, há vários instrumentos de avaliação do aspecto fonológico da linguagem, como, por exemplo, o Clinical Assessment of Articulation and Phonology - CAAP(11), o Test para Evaluar Procesos de Simplificación Fonológica - TEPROSIF-R(12) e o Goldman Fristoe 2 - Test of Articulation - GFTA $2^{(1)}$, que passaram por várias etapas de elaboração e por estudos psicométricos (validade, fidedignidade e normatização) para auxiliar no diagnóstico de crianças com suspeita de desvio fonológico.

No Brasil, os instrumentos mais utilizados são a Avaliação Fonológica da Criança $-\mathrm{AFC}^{(13)}$ e o Teste de Linguagem Infantil
- Fonologia - ABFW ${ }^{(14)}$. Esses dois instrumentos trouxeram importantes contribuições à Fonoaudiologia no que se refere a avaliação e identificação da idade de desenvolvimento fonológico de crianças de duas regiões do Brasil (Sul e Sudeste). No entanto, nenhum deles foi testado quanto a validade e confiabilidade. Passar por estudos psicométricos é fundamental para demonstrar que um instrumento é adequado e que os resultados atingem o objetivo para o qual ele foi desenvolvido.

Assim, a carência de instrumentos elaborados com critérios psicométricos pode refletir na definição de condutas terapêuticas, na elaboração de planos de intervenção ${ }^{(15)} \mathrm{e}$, até mesmo, nas reavaliações para o seguimento ou não da terapia. Portanto, é fundamental a construção e/ou adaptação de instrumentos clínicos que passem por etapas bem definidas e procedimentos rigorosos, bem como apresentem estudos psicométricos para que sua utilização seja válida, confiável e replicável ${ }^{(16-20)}$. Devido à necessidade de instrumentos que tenham passado por estudos psicométricos, foi elaborado e testado o Instrumento de Avaliação Fonológica - INFONO ${ }^{(21)}$.

\section{Instrumento de Avaliação Fonológica: INFONO ${ }^{(21)}$}

O INFONO ${ }^{(21)}$ foi desenvolvido em software para avaliação do desvio fonológico. O objetivo deste instrumento em software é facilitar a avaliação fonológica e disponibilizar ao fonoaudiólogo um instrumento de aplicação simples e rápida, além de ser mais atrativo e interessante para as crianças.

Para a construção deste instrumento, foram realizadas algumas etapas: (1) revisão de literatura e seleção de palavras de estímulo; (2) análise de validade de conteúdo por juízes especialistas; (3) análise de validade de conteúdo por juízes não especialistas (crianças), e (4) estudo piloto. O estudo piloto será apresentado nesse artigo.

Etapa 1 - Revisão de literatura e seleção de palavras de estímulo: foram selecionadas palavras de estímulo a partir dos testes disponíveis no PB e uma lista expressiva de vocabulário, que resultou em um conjunto de itens em potencial para comporem palavras estímulos de um teste de avaliação fonológica. Cada item deveria corresponder a uma imagem que as crianças poderiam facilmente identificar ${ }^{(2)}$.

Etapa 2 - Análise da validade de conteúdo por juízes especialistas: esta etapa envolveu 11 juízes (cinco Fonoaudiólogos Doutores, três Linguistas Doutores e três Fonoaudiólogos Clínicos). Estes juízes foram solicitados a selecionar as melhores palavras de estímulo da lista criada na Etapa 1, com base em seu conhecimento teórico e clínico ${ }^{(22)}$. As palavras selecionadas nesta etapa foram então representadas por imagens e apresentadas aos juízes não especialistas (crianças).

Etapa 3 - Análise da validade de conteúdo por juízes não especialistas: essa etapa envolveu 72 crianças com desenvolvimento fonológico típico, as quais foram solicitadas a avaliar sua familiaridade com os itens selecionados ${ }^{(22)}$. Nesta etapa, as crianças foram questionadas se conheciam ou não a imagem. A resposta foi classificada de acordo com os seguintes critérios: não familiar (não conhece o item); mais ou menos familiar (conhece o item, não sabe o nome, mas sabe o que faz/para que é usada), e familiar (conhece o item, sabe o nome e para que serve). Depois de avaliar a familiaridade de cada 
item, pediu-se às crianças que nomeassem os itens, usando a seguinte dica do avaliador: "O que é isso?". Sua resposta foi classificada em uma das quatro categorias: eliciou a palavra de estímulo correta; eliciou uma palavra semelhante (sinônimo); eliciou uma palavra incorreta, e não respondeu.

Após a identificação e classificação das imagens pelos juízes não especialistas, foi possível definir quais itens permaneceriam no instrumento. O conjunto final de itens foi selecionado com base nas respostas dos juízes não especialistas, bem como na necessidade de assegurar que cada fonema ocorresse com frequência aproximadamente igual em diferentes posições de palavras e sílabas. Alguns itens precisaram ser adaptados para garantir que fossem corretamente identificados. Após esses ajustes, os itens foram testados em uma amostra piloto (apresentada neste artigo).

Estas etapas contribuíram para a obtenção de evidências de validade baseadas no conteúdo. Esta validade se refere a abrangência e representatividade dos itens para envolver tudo o que o teste se propõe a medir ${ }^{(17)}$. No caso do INFONO ${ }^{(21)}$, a abrangência e a representatividade referem-se à fonologia, ou seja, o instrumento deve apresentar itens que possibilitem avaliar todos os fonemas do PB em todas as posições da sílaba e da palavra, e em mais de uma possibilidade de ocorrência. E, ainda, estes itens devem ser de fácil acesso lexical para a criança.

Dessa forma, o objetivo deste estudo foi analisar a adequação dos itens propostos para o INFONO ${ }^{(21)}$ quanto ao reconhecimento e à produção da palavra-alvo, bem como analisar a consistência interna dos itens selecionados e comparar os resultados obtidos conforme as variáveis sexo, tipo de escola e desenvolvimento fonológico (típico e atípico).

\section{MÉTODO}

Trata-se de uma pesquisa quantitativa, prospectiva, descritiva e transversal. O estudo foi aprovado no Comitê de Ética em Pesquisa (CEP) de uma instituição de ensino superior, sob o protocolo número 23081.005433/2011-65.

Todas as crianças foram autorizadas a participar do estudo mediante a assinatura do Termo de Consentimento Livre e Esclarecido (TCLE) pelos pais e/ou responsáveis. Além disso, as crianças só foram avaliadas após serem convidadas e assentirem oralmente por sua participação na coleta.

\section{Participantes}

A amostra foi recrutada em escolas públicas e privadas mediante autorização das diretorias das mesmas e consentimento dos pais. Participaram 48 crianças pareadas a partir de uma combinação quanto aos seguintes aspectos: desenvolvimento fonológico ( $\mathrm{n}=26$ típico e $\mathrm{n}=22$ atípico); idade (seis faixas etárias de 3 a 8 anos e 11 meses, $n=8$ em cada faixa); tipo de escola ( $n=24$ provenientes de escolas públicas e $n=24$ de escolas privadas), e sexo ( $\mathrm{n}=24$ meninas e $\mathrm{n}=24$ meninos).

\section{Critérios de Inclusão e Exclusão}

Como critérios de inclusão, as crianças deveriam ter o TCLE assinado pelos pais e/ou responsáveis; assentirem sua participação na pesquisa; serem classificadas quanto ao desenvolvimento fonológico em típico ou atípico, além de satisfazerem as combinações em relação a idade, tipo de escola e sexo.

Dos critérios de exclusão, os participantes não poderiam apresentar: alterações significativas de linguagem e/ou vocabulário; alterações fonéticas (distorção na fala, como interdentalização nos fonemas linguodentais ou ceceio em fonemas fricativos); sinais evidentes (ou queixas) de problemas auditivos (otites de repetição, dificuldade auditiva), neurológicos e/ou psicológicos, nem mesmo poderiam ter sido submetidos a tratamento fonoaudiológico anterior.

\section{Seleção da amostra}

Para as crianças participantes da pesquisa, primeiramente os pais responderam a um questionário com perguntas do tipo: "Seu filho apresenta ou apresentou alguma dificuldade para ouvir?"; "Seu filho apresenta ou apresentou alguma dificuldade para produzir ou compreender a fala? Explique."; "Frequenta ou frequentou algum atendimento (fonoaudiológico, neurológico, psicológico, etc.)?", entre outras. Os professores também responderam a um questionário em relação ao comportamento da criança em sala de aula, seu rendimento escolar e se a mesma demonstrava alguma dificuldade para ouvir, enxergar, produzir ou compreender a fala.

Após analisar os questionários, duas fonoaudiólogas doutorandas e experientes na área do estudo realizaram uma conversa informal com a criança, com duração de aproximadamente 15 minutos, a fim de verificar se ela apresentava alterações na linguagem e fala, especialmente omissões e substituições de sons. Em tempo real, essas alterações foram anotadas e posteriormente foram utilizadas para a definição do diagnóstico considerando o esperado de produção dos sons de acordo com a idade ${ }^{(4)}$. Assim, utilizou-se o julgamento clínico do fonoaudiólogo para a inclusão da criança no grupo com desenvolvimento fonológico típico ou atípico.

A amostra ainda foi classificada quanto à idade, ao tipo de escola e ao sexo. Durante esse procedimento, também foi possível observar a presença de alguma alteração fonética, como distorções na fala - por exemplo, interdentalização -, bem como possíveis alterações de vocabulário e/ou linguagem.

A descrição da amostra é apresentada na Tabela 1.

Tabela 1. Caracterização dos participantes quanto ao desenvolvimento fonológico, idade, tipo de escola e sexo

\begin{tabular}{|c|c|c|c|c|c|c|c|}
\hline \multicolumn{2}{|c|}{ Idade } & $\begin{array}{c}3.0-3.11 \\
(n=8)\end{array}$ & $\begin{array}{c}4.0-4.11 \\
(n=8)\end{array}$ & $\begin{array}{c}5.0-5.11 \\
(n=8)\end{array}$ & $\begin{array}{c}6.0-6.11 \\
(n=8)\end{array}$ & $\begin{array}{c}7.0-7.11 \\
(n=8)\end{array}$ & $\begin{array}{c}8.0-8.11 \\
(n=8)\end{array}$ \\
\hline \multirow{2}{*}{ DFT } & $\begin{array}{l}\text { Pública } \\
(\mathrm{M} / \mathrm{F})\end{array}$ & $1 / 1$ & $1 / 1$ & $1 / 1$ & $1 / 1$ & $1 / 1$ & $1 / 1$ \\
\hline & $\begin{array}{l}\text { Privada } \\
(\mathrm{M} / \mathrm{F})\end{array}$ & $1 / 1$ & $1 / 1$ & $1 / 1$ & $1 / 1$ & $1 / 2$ & $1 / 2$ \\
\hline \multirow{2}{*}{ DFA } & $\begin{array}{l}\text { Pública } \\
\text { (M/F) }\end{array}$ & $1 / 1$ & $1 / 1$ & $1 / 1$ & $1 / 1$ & $1 / 1$ & $1 / 1$ \\
\hline & $\begin{array}{l}\text { Privada } \\
(\mathrm{M} / \mathrm{F})\end{array}$ & $1 / 1$ & $1 / 1$ & $1 / 1$ & $1 / 1$ & $1 / 0$ & $1 / 0$ \\
\hline
\end{tabular}
Atípico; $M$ = masculino; $F=$ feminino

Nas idades de 7 e 8 anos, não foram captados participantes de escola privada do sexo feminino com desenvolvimento 
fonológico atípico, permanecendo dois com desenvolvimento fonológico típico. A combinação "tipo de escola" foi inserida com o intuito de abranger crianças com diferentes realidades sociais, embora, neste estudo, este dado não tenha sido analisado separadamente.

\section{Material}

O material utilizado para a coleta de dados foi uma versão Beta do software INFONO ${ }^{(21)}$ desenvolvido para avaliar crianças a partir dos 3 anos de idade. A versão Beta de um software é a versão em desenvolvimento do mesmo, mas que é considerada aceitável para uso, mesmo que ainda possua problemas que precisarão ser reparados pelos desenvolvedores antes do lançamento definitivo da sua versão final. Essas versões auxiliam na eliminação de problemas de forma antecipada.

O INFONO ${ }^{(21)}$, versão Beta, foi composto por 116 itens que deveriam ser nomeados espontaneamente pelas crianças. Esses itens forneciam três ou mais oportunidades de produção de cada fonema do PB, em diferentes posições na sílaba/palavra. Os itens selecionados variavam quanto a posição do fonema, tonicidade e número de sílabas, a fim de equilibrar as dificuldades do teste.

Para cada item, o INFONO ${ }^{(21)}$ fornece uma pergunta-chave para ser realizada pelo avaliador, como, por exemplo, " $O$ menino vai dar um...?" (beijo), "Ele vai usar o lápis para...?" (escrever), "Que bicho é este?" (cavalo), etc. A pergunta-chave junto com a imagem direciona para a produção da palavra-alvo desejada para cada item. Se necessário, outras perguntas podem ser utilizadas a fim de obter a palavra-alvo do item desejado.

Esta versão Beta do software apresentava opções de marcação de acordo com a produção do item pela criança, como: eliciou corretamente; eliciou com alterações; eliciou palavras similares, e não eliciou. A opção "eliciou corretamente" foi marcada quando a criança produziu o alvo corretamente sem omissão e/ou substituição de fonema; a opção "eliciou com alterações" foi selecionada quando a criança falou o alvo desejado, mas com presença de omissão e/ou substituição de fonema. A opção "eliciou palavras similares" foi selecionada quando a criança falou outra palavra relacionada ao item e, neste caso, anotavase ao lado a palavra produzida pela criança, e a opção "não eliciou" foi marcada quando a criança não produziu o alvo e/ ou produziu outras palavras não relacionadas ao item.

No momento da avaliação, a criança nomeou o item e o avaliador registrou a resposta em tempo real no próprio software. Para isso, o software forneceu uma série de opções de transcrição fonética para a palavra-alvo, tanto a correta quanto as alteradas, para serem selecionadas pelo avaliador durante a testagem e tornar a avaliação mais rápida. As transcrições das palavras-alvo de cada item foram elaboradas a partir das possibilidades de produção da fala, tanto típica quanto atípica, descritas na literatura.

O INFONO ${ }^{(21)}$ versão Beta oferecia também a possiblidade de transcrever outras opções de produção não listadas, a partir do teclado virtual que apresenta todos os fonemas do Alfabeto Fonético Internacional (Doulos SIL).

\section{Procedimentos}

A coleta de dados pelo INFONO ${ }^{(21)}$ foi realizada por duas fonoaudiólogas doutorandas e experientes na área do estudo, com treinamento prévio para a utilização do software. A coleta de dados foi realizada somente por nomeação espontânea, a fim de verificar a adequação dos itens do $\operatorname{INFONO(21)~quanto~ao~}$ reconhecimento e à produção destes. A criança visualizava o item na tela do computador e eliciava o nome, e imediatamente o avaliador assinalava uma das opções de marcação da resposta da criança (eliciou corretamente, eliciou com alterações, eliciou palavras similares, e não eliciou).

Durante a avaliação, o avaliador realizou a pergunta-chave fornecida pelo software, como, por exemplo, "O menino vai dar um...?" (beijo). Para alguns itens, a criança produzia o alvo antes mesmo de ser realizada a pergunta. Quando o alvo correto não foi obtido mediante a pergunta realizada, $\mathrm{o}$ avaliador usava outra pergunta, a qual era anotada no campo de observação do software.

Os resultados da avaliação de cada participante no formato HTML foram utilizados para elaborar uma planilha no Excel com as respostas de todas as crianças para os 116 itens analisados. A partir disso, calculou-se a consistência interna para o total dos itens do INFONO ${ }^{(21)}$ (versão Beta) pelo Alfa de Cronbach e o percentual de reconhecimento de cada um dos itens pela frequência de produção da palavra-alvo das opções "eliciou corretamente" e "eliciou com alterações". Considerou-se o percentual de reconhecimento por acreditar que, quando a criança eliciou a palavra-alvo corretamente ou com alterações, foi porque ela reconheceu a imagem, visto que é mais fácil para a criança reconhecer o alvo representado do que eliciar corretamente esse alvo ${ }^{(22)}$.

Em seguida, dividiram-se os itens em listas por fonema e posição na sílaba que estava sendo avaliada, em ordem decrescente do percentual de reconhecimento. A partir disso, foram analisadas as dificuldades no reconhecimento e na produção dos alvos considerando a ordem decrescente de complexidade das estruturas silábicas, com a seguinte ordem: $\left(1 .^{\circ}\right)$ Palavras que apresentavam a estrutura de Onset Complexo (OC); (2..$\left.^{\circ}\right)$ Palavras que avaliam a posição de Coda; $\left(3 .^{\circ}\right)$ Palavras que avaliam a posição de Onset Simples, iniciando pelos fonemas com menor número de possibilidade de ocorrência nas palavras, como o $/ \mathrm{n} /, / \mathrm{z} /, / \mathrm{f} /, / \mathrm{g} /$.

Para a seleção das palavras, foram considerados os seguintes percentuais de reconhecimento: $80 \%$ ou mais, o item permaneceu no instrumento; de $60 \%$ a $79 \%$, a imagem ou a pergunta-chave precisou ser modificada para obter a palavra-alvo; menos de $60 \%$, o item foi eliminado do instrumento. Deu-se preferência para as palavras com maior percentual de reconhecimento. Quando não foi possível obter no mínimo três palavras-alvo para o fonema na mesma posição, considerou-se o percentual de $60 \%$ a $79 \%$, mas este item precisou ser modificado para se tornar adequado, de mais fácil reconhecimento para ser eliciado.

Ainda, se no instrumento houvesse mais de três palavras para avaliar determinado fonema na mesma posição (mesmo os itens com percentuais maiores de $80 \%$ ), estas foram eliminadas com o objetivo de diminuir o número de itens de avaliação do 
INFONO ${ }^{(21)}$, primando pela proporção equilibrada destes, ou seja, evitando a super-representação de um fonema e a subrepresentação de outro. A exclusão destes itens foi criteriosa quanto a não prejudicar a ocorrência mínima de três possibilidades do fonema na mesma posição ou de outros fonemas menos frequentes no instrumento. Dessa forma, foi possível obter um número mínimo de itens adequados para permanecerem no INFONO(21), sendo novamente calculada a consistência interna para o total dos itens (versão final).

\section{Análise dos Dados}

Foi realizada uma análise descritiva das respostas das crianças do estudo piloto. Analisou-se a adequação dos itens do INFONO ${ }^{(21)}$ pelo percentual de reconhecimento das imagens e pela produção das palavras-alvo. Isso permitiu averiguar as dificuldades de identificação do objeto representado nas imagens, a familiaridade na produção das palavras-alvo, a adequação das perguntas-chave e, ainda, a consistência interna para do total de itens para o desenvolvimento do INFONO ${ }^{(21)}$, tanto para a versão Beta quanto para a versão final.

O percentual de reconhecimento das imagens foi calculado pela frequência de respostas das opções "eliciou corretamente" e "eliciou com alterações", para cada item. Foi realizada a análise de consistência interna dos itens por meio do Alfa de Cronbach. Em seguida, pontuou-se cada item que permaneceu no INFONO $^{(21)}$ da seguinte forma: 0 , não eliciou o item; 1 , eliciou um item similar; 2 , reconheceu e eliciou o item com troca com alterações, e 3, reconheceu e eliciou o item corretamente. Depois, calculou-se a média de desempenho de produção de cada item para, então, comparar os resultados obtidos conforme o sexo, o tipo de escola e o tipo de desenvolvimento fonológico. Utilizouse para isso o Teste $U$ de Mann-Whitney. Consideraram-se resultados significativos quando $p<0,05$. O pacote estatístico utilizado foi o SPSS versão 22 para Windows.

\section{RESULTADOS}

No geral, o índice de reconhecimento encontrado foi alto para a maioria dos itens, isto é, superior a $80 \%$ em 93 itens $(80,2 \%)$ testados, sendo que, destes, 69 itens $(74,2 \%)$ tiveram um índice superior a 90\% de acertos. Entre 60 e $79 \%$, foram obtidos 16 itens, e, menor que $60 \%$, apenas sete itens. Para melhor compreensão, as tabelas de resultados são apresentadas por ordem decrescente de complexidade da estrutura silábica, pois os itens foram analisados nessa ordem (OC, Coda, Onset). Ainda, nas tabelas, os itens foram divididos por fonemas e por posição na sílaba e na palavra.

Em relação à estrutura de $\mathrm{OC}$ (Tabela 2), manteve-se o máximo possível de itens considerando os percentuais. Foram excluídos do instrumento apenas os itens que apresentaram um percentual muito baixo de reconhecimento (menor que $60 \%$ ), como, por exemplo, para o/bl/ (blusa, blusão), /kl/ (clara, classe) e/gl/ (glacê). Para estes, não foi possível obter alvos que fossem compatíveis com o vocabulário infantil na posição de OI. Essa dificuldade reside, principalmente, em conseguir palavras-alvo representáveis por meio de imagens e que pertençam ao léxico de crianças pequenas. Para outros encontros consonantais, foi possível obter apenas uma palavra, como, por exemplo, $\mathrm{o} / \mathrm{vr} /$ (livro), que só foi selecionado este em $\mathrm{OC}$, sendo que este encontro consonantal não é possível ocorrer na estrutura silábica de OI no PB.

Tabela 2. Percentual de reconhecimento dos itens que avaliam o Onset Complexo

\begin{tabular}{|c|c|c|c|c|}
\hline \multirow{2}{*}{$\begin{array}{l}\text { Onset } \\
\text { Complexo }\end{array}$} & Palavras & $\begin{array}{c}\% \text { de } \\
\text { reconhecimento }\end{array}$ & Palavras & $\begin{array}{c}\% \text { de } \\
\text { reconhecimento }\end{array}$ \\
\hline & Ol & $\%(n)$ & OM & $\%(n)$ \\
\hline \multirow{2}{*}{$/ \mathrm{pr} /$} & prato & $100(48)$ & soprar & 91,67 (44) \\
\hline & presente & $97,92(47)$ & & \\
\hline \multirow{2}{*}{$/ \mathrm{pl} /$} & plástico & $72,92(35)$ & - & - \\
\hline & placa* $^{*}$ & $64,58(31)$ & - & - \\
\hline \multirow{3}{*}{ /br/ } & brinco & $97,92(47)$ & cobra & 91,67 (44) \\
\hline & bruxa & $95,83(46)$ & zebra & $87,50(42)$ \\
\hline & branco & 91,67 (44) & & \\
\hline \multirow{2}{*}{ /bl/ } & blusão & $35,42(17)$ & biblioteca* & 68,75 (33) \\
\hline & blusa & $25,00(12)$ & bíblia & 31,25 (15) \\
\hline \multirow{2}{*}{$/ \mathrm{tr} /$} & trem & $100(48)$ & estrela & $95,83(46)$ \\
\hline & travesseiro & $87,50(42)$ & letra & $85,42(41)$ \\
\hline \multirow{2}{*}{$/ \mathrm{dr} /$} & dragão & $85,42(41)$ & pedra & 93,75 (45) \\
\hline & & & vidro* & 79,17 (38) \\
\hline \multirow{2}{*}{$/ \mathrm{kr} /$} & cruz $^{*}$ & $64,58(31)$ & escrever & $83,33(40)$ \\
\hline & crocodilo & $64,58(31)$ & microfone & 81,25 (39) \\
\hline \multirow{2}{*}{$/ \mathrm{kl} /$} & clara & $37,50(18)$ & bicicleta & $93,75(45)$ \\
\hline & classe & 25,00 (12) & chiclete $^{\star}$ & $62,50(30)$ \\
\hline \multirow{2}{*}{$/ g r /$} & grama & $87,50(42)$ & magro & $87,50(42)$ \\
\hline & gritar* & 66,67 (32) & igreja* & 72,92 (35) \\
\hline$/ g \mid /$ & glacê & $4,17(2)$ & - & - \\
\hline \multirow{2}{*}{$/ \mathrm{fr} /$} & fralda & $89,58(42)$ & refrì $^{\star}$ & 77,08 (37) \\
\hline & fruta & $85,42(41)$ & chifre $^{\star}$ & $64,58(31)$ \\
\hline \multirow{2}{*}{$/ f \mathrm{l} /$} & flor & $97,92(47)$ & - & - \\
\hline & floresta* & 66,67 (32) & - & - \\
\hline$/ \mathrm{vr} /$ & - & - & livro & $97,92(47)$ \\
\hline
\end{tabular}

Legenda: $\mathrm{OI}=$ Onset Inicial; $\mathrm{OM}=$ Onset Medial; negrito = palavras que permaneceram no INFONO

* necessidade de adequar o item; - sem possibilidade de palavras para crianças na faixa etária estudada

Quanto à estrutura de Coda (Tabela 3), verificou-se que a maioria dos itens que permaneceram no instrumento apresentou um percentual de reconhecimento maior que $80 \%$, sugerindo a adequação das imagens utilizadas para obter a produção do alvo. No entanto, alguns itens com percentual entre 60 e $79 \%$, como "plástico", "cruz", "floresta" e "gritar", tiveram suas imagens reelaboradas para facilitar a produção do alvo. Todas estes permaneceram no instrumento pela necessidade de avaliar também a estrutura de OC. 
Tabela 3. Percentual de reconhecimento dos itens que avaliam a posição de Coda

\begin{tabular}{llclc}
\hline & $\begin{array}{c}\text { Palavras } \\
\text { CM }\end{array}$ & $\begin{array}{c}\text { \% de } \\
\text { reconhecimento } \\
\%(\boldsymbol{n})\end{array}$ & $\begin{array}{l}\text { Palavras } \\
\text { CF }\end{array}$ & $\begin{array}{c}\% \text { de } \\
\text { reconhecimento } \\
\%(\boldsymbol{n})\end{array}$ \\
\hline Nasal & & & & \\
\hline & língua & $97,92(47)$ & trem & $100(48)$ \\
& presente & $97,92(47)$ & batom & $97,92(47)$ \\
& brinco & $97,92(47)$ & nuvem & $87,50(42)$ \\
$/ \mathrm{N} / \star \star$ & dente & $91,67(44)$ & & \\
& ventilador & $91,67(44)$ & & \\
& branco & $91,67(44)$ & & \\
& laranja & $83,33(40)$ & & \\
\hline
\end{tabular}

\begin{tabular}{lllll}
\hline Fricativa & & & & \\
\hline & escada & $100(48)$ & óculos & $100(48)$ \\
& espelho & $97,92(47)$ & lápis & $95,83(46)$ \\
& estrela & $95,83(46)$ & tênis & $95,83(46)$ \\
/S/ & pastel & $89,58(43)$ & nariz & $87,50(42)$ \\
& escrever & $83,33(40)$ & cruz $^{*}$ & $64,58(31)$ \\
& plástico* $^{*}$ & $72,92(35)$ & & \\
floresta* $^{*}$ & $66,67(32)$ & & \\
\hline
\end{tabular}

\begin{tabular}{cccll}
\hline \multicolumn{1}{l}{ Líquidas } & & & \\
\hline & porta & $100(48)$ & flor & $97,92(47)$ \\
& porco & $97,92(47)$ & colher & $97,92(47)$ \\
& garfo & $95,83(46)$ & ventilador & $91,67(44)$ \\
$/ \mathrm{r} / \quad$ jornal & $83,33(40)$ & soprar & $91,67(44)$ \\
& & & escrever & $83,33(40)$ \\
& & & gritar* & $66,67(32)$ \\
& & & zíper & $33,33(16)$ \\
\hline & & & anel & $89,58(43)$ \\
& calça & $100(48)$ & pastel & $89,58(43)$ \\
& bolsa & $97,92(47)$ & jornal & $83,33(40)$ \\
\hline
\end{tabular}

Legenda: $\mathrm{CM}=$ Coda Medial; $\mathrm{CF}=$ Coda Final; negrito = palavras que permaneceram no INFONO

${ }^{\star}$ necessidade de adequar o item; ${ }^{\star \star}$ fonema nesta posição é marcado pela nasalidade; ${ }^{\star \star \star}$ fonema nesta posição é produzido como uma semivogal

Em relação à estrutura de Onset (Tabela 4), observou-se que a maioria das imagens correspondentes às palavras-alvo apresentou um percentual de reconhecimento maior que $80 \%$, sugerindo que os itens estavam adequados para se obter a produção da palavra-alvo desejada. Ainda, constatou-se que os percentuais mais baixos de reconhecimento dos itens estavam relacionados com os que avaliam também as estruturas de $\mathrm{OC}$ e Coda. As imagens correspondentes às palavras-alvo "biblioteca", "beijo", "cruz", "chifre", "terra", "plástico", "gritar", "floresta", "chiclete", "placa", "vidro", "zero", "refri”, "igreja"e "navio" que permaneceram no instrumento foram reelaboradas para facilitar a produção do alvo. Todas essas palavras-alvo permaneceram no instrumento pela necessidade de avaliar a estrutura de OC, com exceção de "zero", "beijo", "terra" e "navio".
Tabela 4. Percentual de reconhecimento dos itens que avaliam a posição de Onset

\begin{tabular}{|c|c|c|c|c|}
\hline & $\begin{array}{l}\text { Palavras } \\
\text { OI }\end{array}$ & $\begin{array}{c}\% \text { de } \\
\text { reconhecimento } \\
\%(n)\end{array}$ & $\begin{array}{l}\text { Palavras } \\
\text { OM }\end{array}$ & $\begin{array}{c}\% \text { de } \\
\text { reconhecimento } \\
\% \text { (n) }\end{array}$ \\
\hline \multicolumn{5}{|c|}{ Nasais } \\
\hline \multirow{5}{*}{$/ \mathrm{m} /$} & mesa & $100(48)$ & cama & $100(48)$ \\
\hline & macaco & $97,92(47)$ & grama & $87,50(42)$ \\
\hline & mão & $97,92(47)$ & caminhão & $87,50(42)$ \\
\hline & magro & $87,50(42)$ & tomate & 81,25 (39) \\
\hline & microfone & 81,25 (39) & & \\
\hline \multirow{7}{*}{$/ \mathrm{n} /$} & nuvem & $87,50(42)$ & chinelo & $100(48)$ \\
\hline & nariz & $87,50(42)$ & banana & $100(48)$ \\
\hline & navio* & 66,67 (32) & panela & $95,83(46)$ \\
\hline & & & tênis & $95,83(46)$ \\
\hline & & & anel & $89,58(43)$ \\
\hline & & & jornal & $83,33(40)$ \\
\hline & & & microfone & $81,25(39)$ \\
\hline \multirow{3}{*}{$/ \mathrm{n} /$} & - & - & galinha & $91,67(44)$ \\
\hline & & & passarinho & 91,67 (44) \\
\hline & & & caminhão & $87,50(42)$ \\
\hline \multicolumn{5}{|c|}{ Plosivas } \\
\hline \multirow{8}{*}{$/ \mathrm{p} /$} & pé & $100(48)$ & sapo & $100(48)$ \\
\hline & porta & $100(48)$ & sapato & $97,92(47)$ \\
\hline & porco & $97,92(47)$ & copo & $97,92(47)$ \\
\hline & panela & $95,83(46)$ & espelho & $97,92(47)$ \\
\hline & pedra & $93,75(45)$ & lápis & $95,83(46)$ \\
\hline & passarinho & 91,67 (44) & tapete & $95,83(46)$ \\
\hline & pastel & $89,58(43)$ & chapéu & $95,83(46)$ \\
\hline & & & zíper & $33,33(16)$ \\
\hline \multirow{10}{*}{ /b/ } & bola & $100(48)$ & cabelo & $95,83(46)$ \\
\hline & banana & $100(48)$ & rabo & $89,58(43)$ \\
\hline & bolsa & $97,92(47)$ & bebê & $87,50(42)$ \\
\hline & batom & $97,92(47)$ & & \\
\hline & bicicleta & $93,75(45)$ & & \\
\hline & barriga & 91,67 (44) & & \\
\hline & bebê & $87,50(42)$ & & \\
\hline & biblioteca* & $68,75(33)$ & & \\
\hline & beijo* $^{\star}$ & $64,58(31)$ & & \\
\hline & bíblia & $15(31,25)$ & & \\
\hline \multirow{18}{*}{$/ \mathrm{t} /$} & tapete & $95,83(46)$ & gato & $100(48)$ \\
\hline & tênis & $95,83(46)$ & prato & $100(48)$ \\
\hline & tesoura & $95,83(46)$ & porta & $100(48)$ \\
\hline & tomate & 81,25 (39) & sapato & $97,92(47)$ \\
\hline & terra* & $68,75(33)$ & presente & $97,92(47)$ \\
\hline & & & batom & $97,92(47)$ \\
\hline & & & tapete & $95,83(46)$ \\
\hline & & & bicicleta & $93,75(45)$ \\
\hline & & & ventilador & $91,67(44)$ \\
\hline & & & dente & 91,67 (44) \\
\hline & & & pastel & $89,58(43)$ \\
\hline & & & fruta & $85,42(41)$ \\
\hline & & & tomate & 81,25 (39) \\
\hline & & & plástico* & $72,92(35)$ \\
\hline & & & biblioteca* & $68,75(33)$ \\
\hline & & & gritar $^{\star}$ & 66,67 (32) \\
\hline & & & floresta* & 66,67 (32) \\
\hline & & & chiclete* $^{\star}$ & $62,50(30)$ \\
\hline
\end{tabular}


Tabela 4. Continuação...

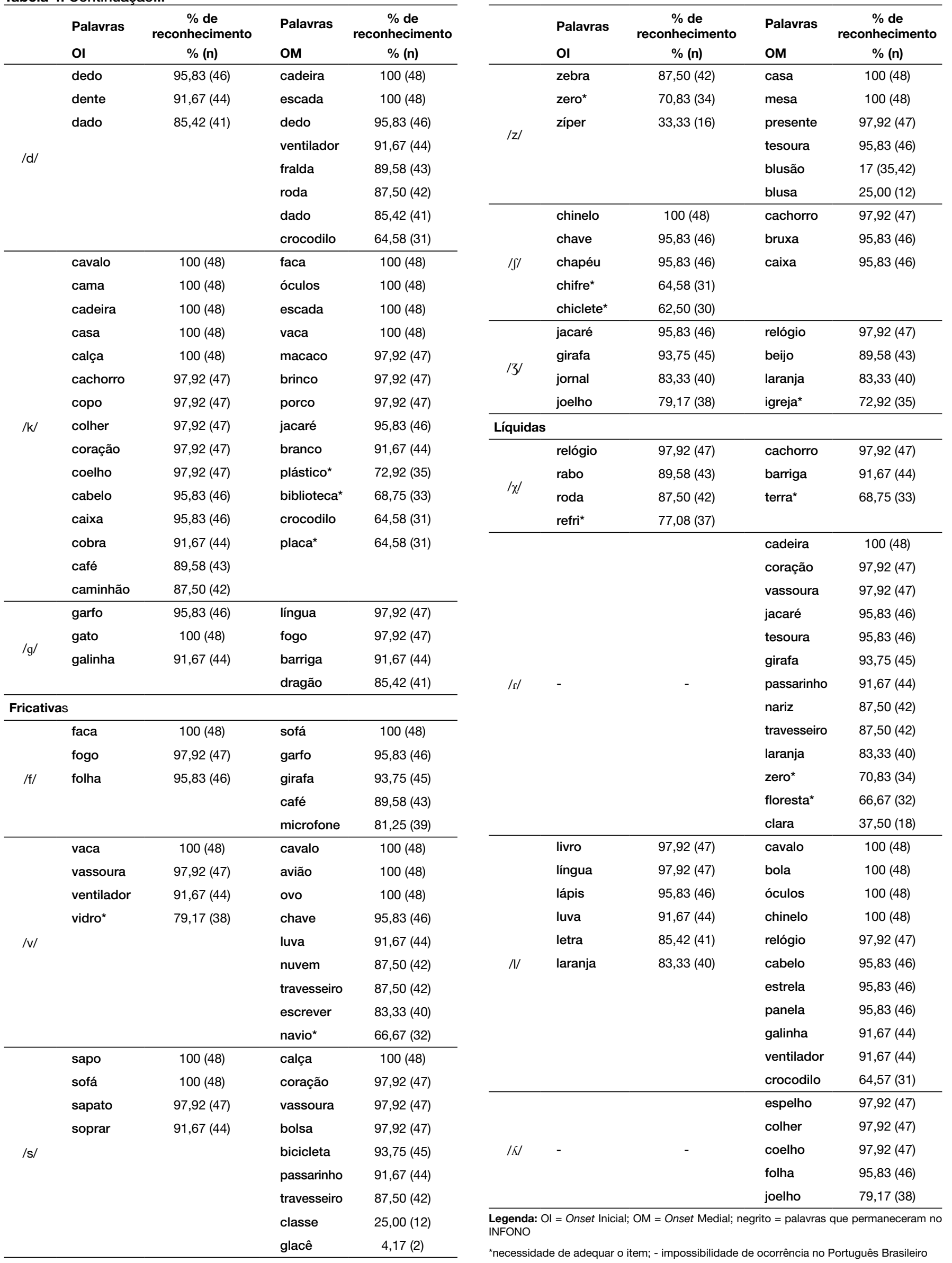


Além da imagem, a pergunta-chave para a palavra-alvo "navio" $(66,67 \%)$ precisou ser adequada. Várias crianças identificavam inicialmente a imagem como "barco". A permanência deste alvo era importante para que ocorressem três possibilidades do fonema /n/ em OI. A imagem de um "navio" precisou ser modificada para um "navio pirata" e, dessa forma, a pergunta-chave que era "O que é isto?" passou a ser "É de pirata, é um ...?".

Ainda, alterações na representação das imagens foram realizadas com base nas anotações realizadas durante as respostas das crianças às perguntas do INFONO ${ }^{(21)}$. Na imagem que representava o alvo "chiclete", muitas crianças identificavam e produziam como "balão". Para obter a produção desse alvo, percebeu-se que o essencial foi tornar a imagem "animada" com [gifs] (termo dado às animações formadas por várias imagens GIF compactadas numa só, dando movimento ao item), ou seja, colocar uma sequência de movimentos: menino mastigando, fazendo uma bolha, a bolha estourando e o chiclete grudado no rosto do menino. O mesmo aconteceu para os alvos "beijo" e "gritar", que depois de se tornarem "animadas", facilitou-se a compreensão do item.

Para o alvo "terra", muitas crianças falavam areia molhada. A imagem foi modificada e, dessa forma, passou a aparecer os movimentos de um menino plantando uma muda de árvore. A pergunta-chave "O que é isto?" passou a ser "O menino está plantando na ...?".

O alvo "vidro" várias vezes foi identificado e produzido como "janela", dificultando a eliciação da palavra-alvo. Assim, a imagem foi modificada para uma pedra quebrando o vidro da janela e a pergunta-chave passou a ser " $A$ pedra quebrou $O$ ...?" em vez de "A janela tem ...?".

Para o alvo "plástico", apenas a pergunta foi reelaborada para "Este copo não é de vidro, é de ...?". A imagem correspondente ao alvo "zero" foi reelaborada, aumentando a sequência de números que era "0 1" para "0 12 2 3", e o número "zero" foi "animado", aumentando de tamanho, pois muitas crianças produziam o numeral ['d $\mathrm{d} s$ ] ou a vogal aberta [ว]. Todos os itens do INFONO(21) foram "animados" com gifs, alguns por necessidade e outros para se tornarem mais atrativos.

Percebeu-se, ainda, que era possível excluir alguns itens sem prejudicar o mínimo de ocorrências (três possibilidades) do fonema na mesma posição ou de outros fonemas no instrumento. Dessa forma, algumas palavras, como "escada", "luva", "tomate", "óculos", "banana”, etc., puderam ser excluídas para minimizar o número de itens do instrumento.

Após as modificações realizadas, o $\mathrm{INFONO}^{(21)}$ ficou composto por 84 palavras-alvos representadas no software por imagens animadas, primando pelo objetivo de o teste de ser rápido e de fácil aplicação. $\mathrm{O}$ tempo médio da avaliação pelo $\mathrm{INFONO}^{(21)}$ foi de aproximadamente 15 minutos. Conforme a análise do Alfa de Cronbach, o instrumento demonstrou excelente consistência interna tanto para a versão com as 116 palavras (versão Beta) $(\alpha=0,98)$ quanto para a versão final do $\mathrm{INFONO}^{(21)}$ com 84 palavras $(\alpha=0,97)$, demonstrando adequado índice de fidedignidade para os itens. A síntese do número de itens para cada fonema que compõem o instrumento por posição, número de sílaba e tonicidade é apresentada na Tabela 5.

Ainda, analisaram-se as médias de desempenho de produção das palavras-alvo quanto às variáveis sexo, tipo de escola e desenvolvimento fonológico (típico e atípico), que estão apresentadas na Tabela 6 . Os resultados obtidos não evidenciam diferença de desempenho entre as crianças agrupadas por sexo e tipo de escola. Isso demonstra não haver impacto dessas variáveis na produção dos itens do $\operatorname{INFONO}^{(21)}$. No entanto, as crianças com desenvolvimento fonológico atípico obtiveram piores resultados do que as típicas em 60 itens $(76,19 \%)$.

Tabela 5. Número de itens que permaneceram no INFONO para cada fonema por posição, número de sílabas e tonicidade

\begin{tabular}{|c|c|c|c|c|c|c|c|c|c|c|c|c|c|c|c|c|c|c|c|}
\hline \multicolumn{20}{|c|}{ Número de itens $\times$ Posição } \\
\hline & $/ \mathrm{m} /$ & $/ \mathrm{n} /$ & $/ \mathrm{n} /$ & $/ \mathrm{p} /$ & /b/ & $/ \mathrm{t} /$ & $/ d /$ & $/ \mathrm{k} /$ & /g/ & $/ \mathrm{f} /$ & /v/ & /s/ & $|z|$ & $/ \mathrm{d} /$ & /3/ & /l/ & $/ \lambda /$ & $|\chi|$ & $/ \mathrm{d} /$ \\
\hline OI & 3 & 3 & - & 4 & 7 & 3 & 3 & 11 & 3 & 3 & 3 & 4 & 2 & 4 & 3 & 4 & - & 3 & - \\
\hline OM & 3 & 5 & 3 & 5 & 3 & 16 & 4 & 7 & 4 & 4 & 5 & 5 & 4 & 3 & 3 & 7 & 4 & 3 & 8 \\
\hline $\mathrm{CM}$ & - & 5 & - & - & - & - & - & - & - & - & - & 6 & - & - & - & 3 & - & - & 3 \\
\hline CF & - & 3 & - & - & - & - & - & - & - & - & - & 4 & - & - & - & 3 & - & - & 6 \\
\hline \multicolumn{20}{|c|}{ Número de itens $\times$ Número de sílabas } \\
\hline & $/ \mathrm{m} /$ & $/ \mathrm{n} /$ & $/ \mathrm{p} /$ & $/ p /$ & $/ \mathrm{b} /$ & $/ \mathrm{t} /$ & $/ d /$ & $/ \mathrm{k} /$ & /g/ & $/ f /$ & $/ \mathrm{v} /$ & /s/ & $|z|$ & $/ \mathrm{d} /$ & /3/ & /l/ & $/ \lambda$ & $|\chi|$ & $/ \mathrm{d} /$ \\
\hline Monossílabas & 1 & 1 & - & 1 & 0 & 0 & 0 & 0 & 0 & 0 & 0 & 1 & 0 & 0 & 0 & 0 & - & 0 & 1 \\
\hline Dissílabas & 4 & 11 & 0 & 5 & 6 & 10 & 4 & 10 & 5 & 5 & 4 & 9 & 4 & 4 & 2 & 10 & 2 & 3 & 8 \\
\hline Trissílabas & 1 & 2 & 2 & 2 & 2 & 6 & 0 & 7 & 2 & 1 & 2 & 6 & 2 & 3 & 4 & 6 & 2 & 3 & 5 \\
\hline Polissílabas & 1 & 2 & 3 & 1 & 2 & 3 & 1 & 1 & 0 & 1 & 2 & 3 & 0 & 0 & 0 & 1 & 0 & 0 & 3 \\
\hline \multicolumn{20}{|c|}{ Número de itens $\times$ Tonicidade } \\
\hline & $/ \mathrm{m} /$ & $/ \mathrm{n} /$ & $/ \mathrm{n} /$ & $/ p /$ & $/ \mathrm{b} /$ & $/ \mathrm{t} /$ & $/ d /$ & $/ \mathrm{k} /$ & /g/ & $/ f /$ & /v/ & /s/ & /z/ & $/ \int /$ & /3/ & /l/ & $/ \lambda$ & $|\chi|$ & $/ \mathrm{s} /$ \\
\hline Tônica & 2 & 9 & 1 & 5 & 4 & 7 & 4 & 5 & 3 & 5 & 5 & 3 & 4 & 2 & 0 & 12 & 1 & 2 & 13 \\
\hline Átona & 4 & 5 & 2 & 4 & 5 & 12 & 1 & 13 & 4 & 2 & 3 & 16 & 2 & 5 & 6 & 5 & 3 & 4 & 4 \\
\hline
\end{tabular}

Legenda: $\mathrm{OI}=$ Onset Inicial; $\mathrm{OM}=$ Onset Medial; $\mathrm{CM}=$ Coda Medial; CF = Coda Final 
Tabela 6. Comparação entre as médias de desempenho de produção dos itens na versão final do INFONO, de acordo com sexo, tipo de escola e desenvolvimento fonológico

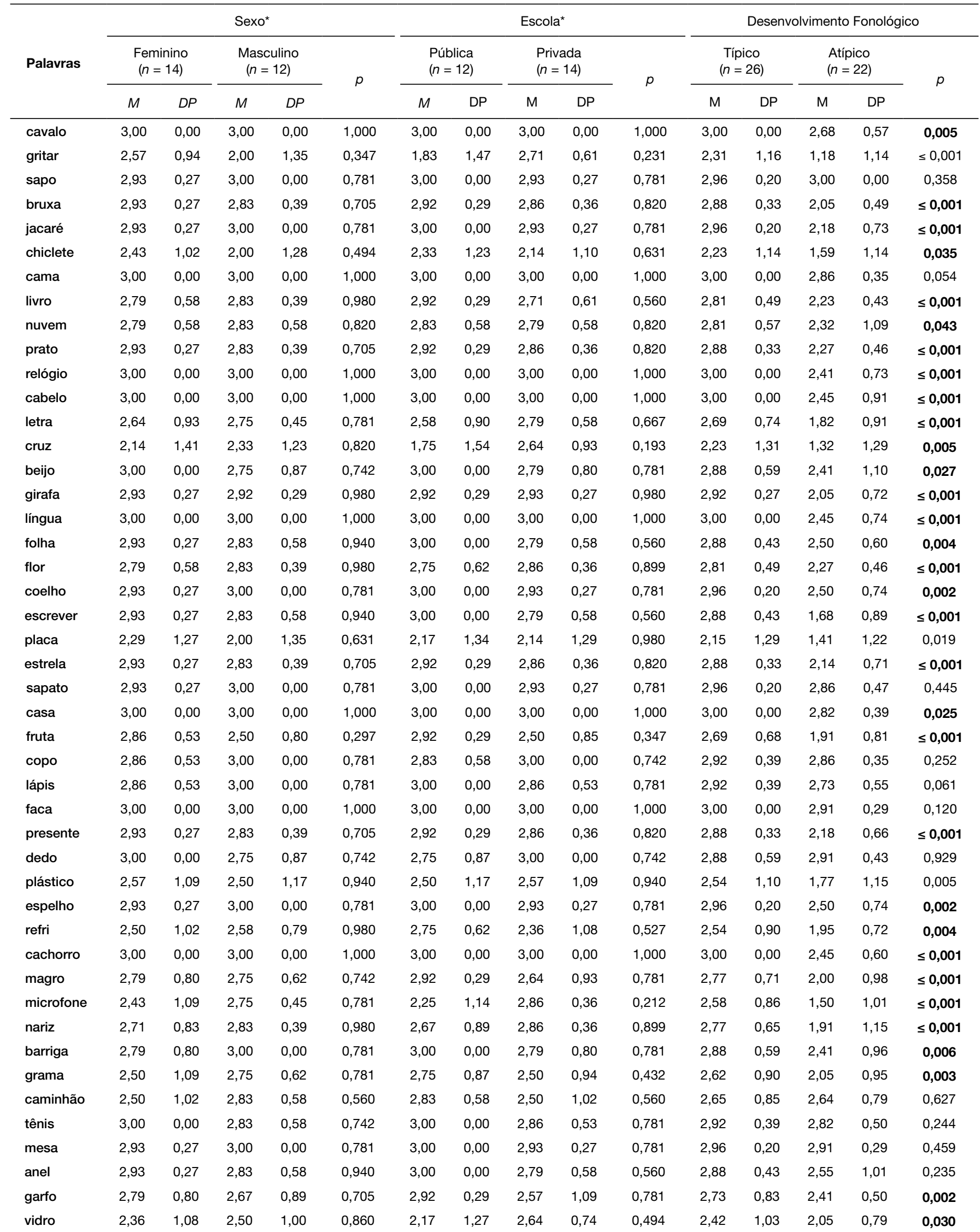


Tabela 6. Continuação...

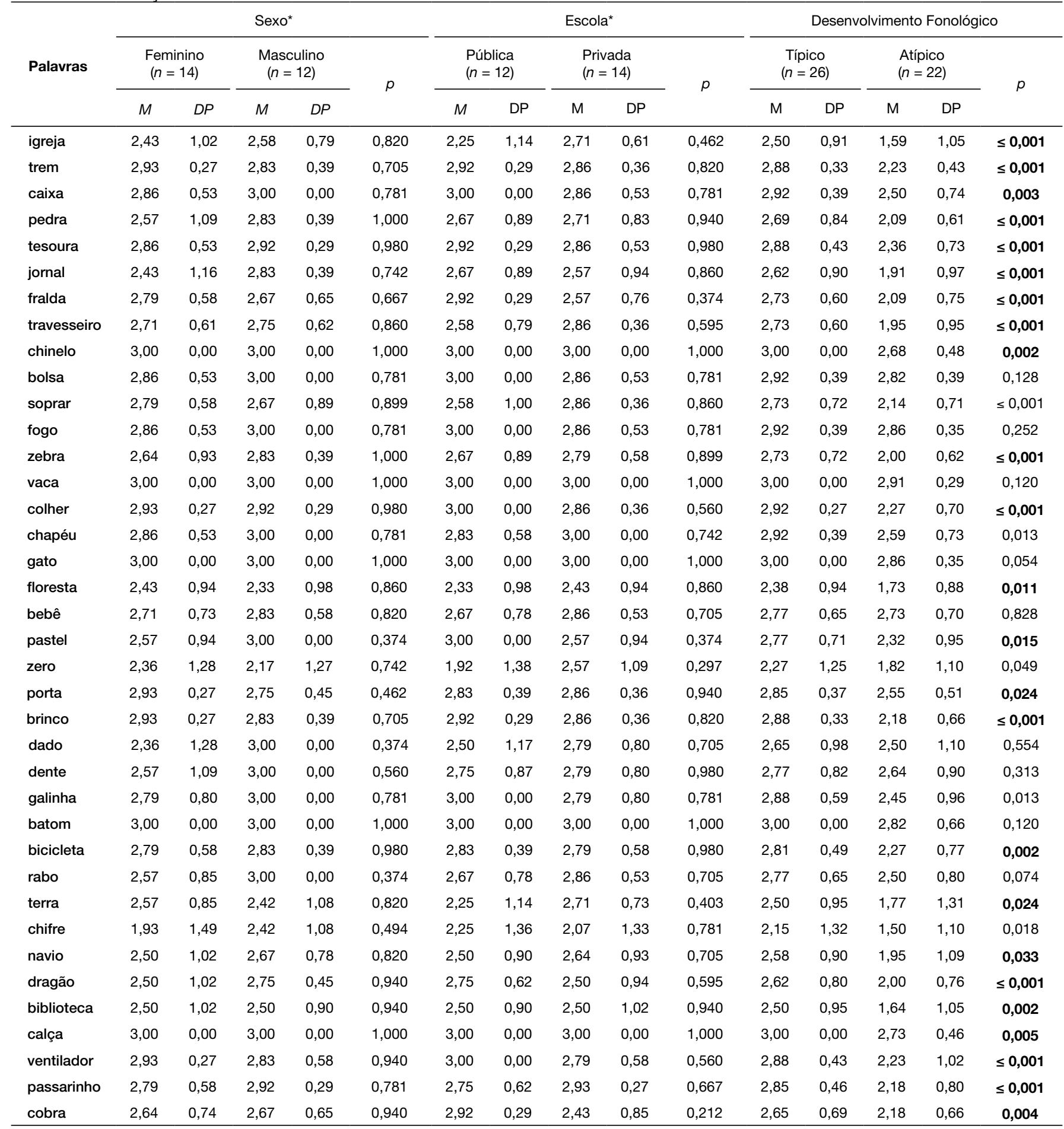

Legenda: *Na comparação de desempenho entre os grupos conforme sexo e tipo de escola, foram incluídas apenas as crianças com aquisição típica

\section{DISCUSSÃO}

A consistência interna verificada foi excelente para o total dos itens, bem como o índice de reconhecimento encontrado foi alto para a maioria dos itens. Isso evidencia que os itens e as imagens estavam adequados para compor o $\mathrm{INFONO}^{(21)} \mathrm{A}$ produção exata da palavra-alvo é importante para não deixar de avaliar e/ou oferecer menos possibilidades de produção para algum fonema em alguma posição na sílaba e/ou palavra.

Para todas as etapas de elaboração de uma ferramenta de avaliação, deve-se indicar claramente sua finalidade e o públicoalvo a que se destina; avaliar a adequação dos itens selecionados; apontar o tipo de palavras incluídas e os métodos utilizados para seleção das palavras-alvo ${ }^{(16,18)}$. Quanto aos itens, deve-se 
ter alguns cuidados, como: usar palavras que são comumente conhecidas por crianças pequenas ou usar alvos que fazem parte da cultura nacional, sendo importante considerar as diferenças regionais, dentre outros ${ }^{(1)}$.

Estes itens são importantes para um instrumento, pois asseguram a precisão diagnóstica, assim como determinam a sensibilidade (identificação precisa da dificuldade da criança) e a especificidade (identificação precisa da ausência de dificuldade em crianças que não apresentam desenvolvimento atípico) do instrumento $^{(16,18)}$.

Uma avaliação precisa é uma das principais ferramentas para o clínico obter um diagnóstico confiável e possibilitar o planejamento adequado da intervenção. Por isso, o INFONO(21) foi elaborado em software para fornecer uma amostra de fala completa, incluindo, no mínimo, três possibilidades de ocorrência para a produção de todas as consoantes do PB, nas diferentes posições que ocorrem na sílaba e na palavra.

Pelos percentuais de reconhecimento dos itens, foi possível verificar se os itens pertenciam ao vocabulário infantil, pois, para nomear, a criança precisa identificar a imagem ou a ação representada. $O$ processo de nomeação por confrontação visual passa por três estágios: (1) identificação do objeto representado; (2) acesso à representação semântica, permitindo que o objeto seja reconhecido, e (3) lexicalização, ou ativação da representação fonológica, por meio da qual o nome da imagem é recuperado e pronunciado ${ }^{(23)}$. Há uma relação entre o desenvolvimento lexical e o inventário fonológico das crianças, sendo importante a realização de pesquisas que mostrem como os fatores lexicais e fonológicos podem influenciar a produção da fala, e como esta informação pode ser utilizada no diagnóstico e tratamento de crianças com desenvolvimento fonológico atípico ${ }^{(24)}$.

Oferecer, no mínimo, três possibilidades de produção de cada fonema do PB em cada posição em que ocorre e duas possibilidades para cada grupo consonantal na estrutura de $\mathrm{OC}$ eram metas para o INFONO ${ }^{(21)}$. No entanto, durante o processo de elaboração, não foi possível atingir esta meta para o fonema /z/ em OI e para alguns OC (/kl/, /kr/, /gl/, /bl/), devido ao baixo percentual de reconhecimento apresentado. Acredita-se que isso pode ter ocorrido por três motivos: pelos itens não pertencerem ao vocabulário de crianças pequenas ou pela dificuldade de representação da palavra-alvo, de modo que fosse reconhecida e produzida pelas crianças ${ }^{(22,25)}$. Ou ainda pelo fato de que o próprio léxico da língua ser restrito quanto a essas possibilidades fonológicas, o que reduz a possibilidade de palavras com essas estruturas serem encontradas na língua e, ainda em menor grau, no vocabulário infantil.

Mesmo assim, verificou-se que o INFONO $^{(21)}$ avalia todos os fonemas do PB em todas as posições da sílaba e da palavra, ou seja, os alvos que permaneceram no instrumento foram suficientes para a compilação do inventário fonético e fonológico da criança. Este aspecto é considerado importante para um instrumento que avalia a fala pela nomeação espontânea de palavras, uma vez que, para concluir com precisão que um som não produzido pela criança está ausente no seu inventário, ela deve ter oportunidades suficientes para produzir este som ${ }^{(26,27)}$. Não foram encontradas pesquisas que indicassem quantas ocorrências seriam suficientes para concluir que uma criança consegue ou não produzir determinado som. Porém, com base no critério de que os sons, para serem atribuídos ao inventário fonético, teriam de ser produzidos de uma a duas vezes, teriam de ser dadas para uma criança, no mínimo, duas oportunidades para a produção de cada fonema ${ }^{(28)}$.

Pela análise, ainda se verificou que alguns fonemas, como, por exemplo, /p/ e /k/ em OI e OM; /b/ em OI; /t/, /r/ e /l/ em $\mathrm{OM}$, apresentavam mais de oito oportunidades de produção, o que seria desnecessário. Com o objetivo de reduzir o número de itens do instrumento, alguns alvos foram excluídos sem que se prejudicasse a ocorrência de outros fonemas de menor ocorrência, como /d/, /g/, /f/, /s/, /z/ em OI ou a avaliação do OC. Mesmo assim, alguns fonemas de grande ocorrência na avaliação permaneceram com mais de três possibilidades, a fim de preservar a presença do $\mathrm{OC}$ e de alguns fonemas de menor ocorrência.

Um estudo $^{(27)}$ analisou 11 testes padronizados para o Inglês com o objetivo de verificar se forneciam itens suficientes para avaliar o inventário consonantal de uma criança. Este estudo observou que os testes foram úteis para identificar crianças com desvio fonológico, porém eles não forneciam o número necessário de oportunidades de produção de fonemas para avaliar todas as consoantes do Inglês. Nesses casos, a avaliação devia ser complementada com sondagens por meio de palavras foneticamente controladas, a fim de determinar os sons que estavam ausentes no inventário fonológico da criança.

Esse mesmo trabalho ${ }^{(27)}$ sugeriu a necessidade de testar fonemas em mais de uma palavra, pelo efeito dos sons antecedentes e seguintes ao fonema a ser avaliado. No caso do $\mathrm{INFONO}^{(21)}$, são oferecidos três ou mais alvos em palavras com diferentes números de sílabas, tonicidade e contexto antecedente e seguinte. Assim, considerou-se importante que o INFONO ${ }^{(21)}$ oferecesse palavras com diferentes números de sílabas (mono, di, tri e polissilábicas) em que o alvo estivesse presente, na medida do possível, tanto em sílaba tônica quanto sílaba átona. Estas variáveis podem influenciar a capacidade de uma criança na produção do alvo ${ }^{(22,27,28)}$.

Testes que não incluem palavras de complexidade variada ${ }^{(27)} \mathrm{ou}$ que incluem somente palavras de alta frequência de uso lexical ${ }^{(26)}$ podem não diagnosticar corretamente o desvio fonológico ou fornecer respostas não reais à capacidade da criança, como em situações cotidianas de fala. Acredita-se que o equilíbrio entre as palavras de um instrumento de avaliação fonológica seja fundamental para obter um resultado fidedigno da avaliação ${ }^{(22)}$.

Ao analisar as palavras-alvo quanto ao sexo, tipo de escola e desenvolvimento fonológico (típica e atípica), observou-se que a produção dos itens lexicais presentes no instrumento não sofreu influência das variáveis sexo e tipo de escola. Apenas na variável tipo de desenvolvimento fonológico, as crianças com desenvolvimento atípico obtiveram pior resultado. Isso era esperado, pois o INFONO ${ }^{(21)}$ foi elaborado para auxiliar o fonoaudiólogo no diagnóstico quanto ao desenvolvimento fonológico típico e atípico. Dessa forma, observa-se que os itens lexicais que permaneceram no instrumento foram precisos na identificação das crianças que apresentavam dificuldades fonológicas. 
Ao encerrar a análise deste estudo piloto, foi possível terminar o desenvolvimento do software $\mathrm{INFONO}^{(21)}$. Porém, esse instrumento ainda deverá passar por outros estudos psicométricos de validade, fidedignidade e normatização. $\mathrm{O}$ instrumento utiliza esses mesmos itens para a obtenção da amostra de fala de três formas: repetição, nomeação espontânea e fala encadeada. Neste estudo-piloto, realizou-se somente a análise a partir da nomeação espontânea, pois considera-se que esta forma de avaliação é vantajosa quanto à possibilidade de coleta de dados. A nomeação espontânea permite avaliar todos os fonemas em todas as posições na sílaba e na palavra. Ainda, não possibilita a imitação da produção do examinador, que pode induzir à produção melhorada da fala. Também, facilita a compreensão da fala da criança pelo examinador, o que pode não ser inteligível na testagem a partir da fala encadeada ${ }^{(27)}$.

\section{CONCLUSÃo}

Este estudo analisou uma situação real de avaliação utilizando o software INFONO ${ }^{(21)}$ versão Beta. Concluiu-se que a maioria das imagens utilizadas permitiu o correto reconhecimento dos itens e, consequentemente, possibilitou a produção do alvo desejado. Poucas imagens precisaram ser reelaboradas para permitir a produção espontânea do alvo. Ainda, obteve-se uma quantidade mínima, mas suficiente, de alvos para compor o instrumento, os quais permitiram uma avaliação eficaz de todo o inventário fonético e fonológico da criança. Assim, evita-se super-representação de um fonema e a sub-representação de outro (proporção equilibrada do número de itens). Além disso, a consistência interna dos itens mostrou-se adequada.

Este instrumento oferece duas ou mais oportunidades de produção para avaliar um mesmo fonema, com o intuito de confirmar se o mesmo está presente ou ausente no inventário fonético; presente, parcialmente adquirido, e ausente no inventário fonológico da criança. Ainda, o INFONO ${ }^{(21)}$ oferece palavras com diferentes números de sílabas em que o alvo está presente, na medida do possível, tanto em sílaba tônica quanto átona.

\section{AGRADECIMENTOS}

Agradecemos à Fundação de Amparo à Pesquisa do Estado do Rio Grande do Sul (FAPERGS), pelo apoio concedido para a realização deste projeto no Edital Pesquisador Gaúcho sob o processo n. ${ }^{\circ} 11 / 2184-8$, e aos bolsistas de Iniciação Científica, que receberam financiamento PROBITI/FAPERGS e PIBITI/ CNPq, ocupadas pelos bolsistas dos cursos de Ciências da Computação-UFSM e Desenho Industrial-UFSM. Além disso, às bolsistas de Iniciação Científica PROBIC/FAPERGS e PROBIC/ CNPq do curso de Fonoaudiologia-UFSM, que auxiliaram na coleta e tabulação dos dados de pesquisa.

\section{REFERÊNCIAS}

1. Goldman R, Fristoe M. Goldman-Fristoe Test of Articulation 2 - Test manual. Circle Pines, MN: American Guidance Services Inc, 2000.

2. Silva MK, Ferrante C, Borsel JV, Pereira MMB. Aquisição fonológica do Português Brasileiro em crianças do Rio de Janeiro. J Soc Bras Fonoaud. 2012;24(3):248-254. https://doi.org/10.1590/S2179-64912012000300010
3. Mezzomo CL, Luiz SW. Interferência da variante linguística nas estratégias de reparo utilizadas no processo de aquisição fonológica. J Soc Bras Fonoaudiol. 2012;24(3):239-247. https://doi.org/10.1590/S2179-64912012000300009

4. Oliveira C, Mezzomo CL, Freitas G, Lamprecht RR. Cronologia da aquisição dos segmentos e das estruturas silábicas; in: Lamprecht RR (eds): Aquisição Fonológica do Português: Perfil de desenvolvimento e subsídios para terapia. Porto Alegre, Artmed, 2004, pp. 167-76

5. Rabelo ATVR, Alves CRL, Goulart LMHF, Friche AADL, Lemos SMA, Campos FR, et al. Alterações de fala em escolares na cidade de Belo Horizonte. J Soc Bras Fonoaud 2011;23(4):344-50. https://doi.org/10.1590/ S2179-64912011000400009

6. Namasivayam AK, Pukonen M, Goshulak D, Yu VY, Kadis DS, Kroll R, et al. Relationship between speech motor control and speech intelligibility in children with speech sound disorders. J Commun Dis 2013;46(3):264-80. https://doi.org/10.1016/j.jcomdis.2013.02.003. PMid:2362822

7. Newmeyer AJ, Grether S, Grasha C, White J, Akers R, Aylward C, et al. Fine motor function and oral-motor imitation skills in preschool-age children with speech-sound disorders. Clin Pediat (Phila) 2007;46(7):604-11. https:// doi.org/10.1177/0009922807299545. PMid:17522288

8. Oshima M, Moret ALM, Amorim RB, Alvarenga KdF, Bevilacqua MC, Lauris JRP, et al. Early Listening Function (ELF): adaptação para a língua portuguesa. Rev Soc Bras Fonoaud 2010;15(2):191. https://doi.org/10.1590/ S1516-80342010000200008

9. Müürsepp I, Aibast H, Gapeyeva H, Pääsuke M. Motor skills, haptic perception and social abilities in children with mild speech disorders. Brain Dev 2012;34(2):128-32. https://doi.org/10.1016/j.braindev.2011.02.002

10. Lousada M, Jesus LMT, Hall JA, Joffe V. Intelligibility as a clinical outcome measure following intervention with children with phonologically basead speech-sound disorders. Int J Lang Commun Disord. 2014;49(5):584-601. https://doi.org/10.1111/1460-6984.12095. PMid:24861159

11. Secord WA, Donohue JS. CAAP - Clinical Assessment of Articulation and Phonology - Supplemental Examiner's Manual. USA: Super Duper®Publications; 2002, 50p

12. Pavez MM, Maggiolo M, Coloma CJ. Test para evaluar procesos de implificación fonológica - TEPROSIF-R. Santiago: Pontificia Univesidad Catolica de Chile, 2009. 83p

13. Yavas M, Hernandorena CLM, Lamprecht RR. Avaliação Fonológica da Criança. Porto Alegre: Artes Médicas; 2002. 148p

14. Wertzner HF. Fonologia (Parte A); in: Andrade CRF, Befi-Lopes DM, Fernades FDM, Wertzner HF. Teste de linguagem infantil nas áreas de fonologia, vocabulário, fluência e pragmática. Carapicuíba: Pró-Fono; 2004

15. Giusti E, Befi-Lopes DM. Tradução e adaptação transcultural de instrumentos estrangeiros para o Português Brasileiro (PB). Pró-Fono 2008;20(3):207-10. https://doi.org/10.1590/S0104-56872008000300012

16. McLeod S, Verdon S. A Review of 30 Speech Assessments in 19 Languages Other Than English. Am J Speech-Lang Path 2014(1):1-16. https://doi org/10.1044/2014_AJSLP-13-0066. PMid:24700105

17. Kirk C, Vigeland L. A Psychometric Review of Norm-Referenced Tests Used to Assess Phonological Error Patterns. Lang Speech Hear Serv Sch 2014;45(4):365-77. https://doi.org/10.1044/2014_LSHSS-13-0053. PMid:25091265

18. Friberg JC. Considerations for test selection: How do validity and reliability impact diagnostic decisions? Child Lang Teach Ther 2010;26(1):77-92. https://doi.org/10.1177/0265659009349972

19. Neves MdB, Borsel JV, Pereira MMdB, Paradela EMP. Cross-cultural adaptation of the Western Aphasia Battery - Revised screening test to Brazilian Portuguese: a preliminary study. CoDAS 2014;26(1):38-45. https://doi.org/10.1590/s2317-17822014000100006

20. Cunha VLO, Capellini SA. Construction and validation of an instrument to assess the reading comprehension of students from the third to the fifth grades of elementary school. CoDAS 2014;26(1):28-37. https://doi. org/10.1590/s2317-17822014000100005

21. Ceron MI. Instrumento de Avaliação Fonológica (INFONO): desenvolvimento e estudos psicométricos. Tese (Doutorado em Distúrbios da Comunicação Humana). Universidade Federal de Santa Maria, Santa Maria 2015 
22. Savoldi A, Ceron MI, Keske-Soares M. Quais são as melhores palavras para compor um instrumento de avaliação fonológica? Audiol Commun Res 2013;8(3):194-202. https://doi.org/10.1590/S2317-64312013000300009

23. Spezzano LC, Mansur LL, Radanovic M. Applicability of the "An Object and Action Naming Battery" in Brazilian Portuguese. CoDAS 2013;25(5):437-43. https://doi.org/10.1590/S2317-17822013000500007

24. Sosa AV, Stoel-Gammon C. Lexical and phonological effects in early word production. J Speech Lang Hear Res 2012;55(2):596-608. https:// doi.org/10.1044/1092-4388(2011/10-0113)

25. Bueno TG, Vidor DCGM, Alves ALSA. Protocolo de avaliação fonológica infantil - PAFI: Projeto piloto. Verba Volant 2010;1(1):53-86

26. Ygual-Fernández A, Cervera-Mérida JF, Rosso P. Utilidad del análisis fonológico en la terapia del lenguaje. Rev Neurol 2008;46(supl 1):S97-S100. https://doi.org/10.33588/rn.46S01.2008009
27. Eisenberg SL, Hitchcock ER. Using standardized tests to inventory consonant and vowel production: a comparison of 11 tests of articulation and phonology. Lang Speech Hear Serv Sch 2010;41(4):488-503. https:// doi.org/10.1044/0161-1461(2009/08-0125). PMid:20581217

28. Keske-Soares M, Pagliarin KC, Ceron MI. Terapia fonológica considerando as variáveis linguísticas. Rev Soc Bras Fonoaudiol 2009;14(2):261-6. https://doi.org/10.1590/S1516-80342009000200019

\section{Contribuição dos autores}

MIC foi responsável pela elaboração do projeto, coleta dos dados, tabulação dos dados, delineamento do estudo, execução do projeto e elaboração do manuscrito; MBG foi responsável pela coleta e colaborou na elaboração do manuscrito; CRO colaborou com a tabulação dos dados, elaboração do manuscrito e foi responsável pela análise estatística dos dados; MKS foi responsável pelo projeto, delineamento do estudo e orientação geral das etapas de execução e revisão do manuscrito. 